\title{
Interculturalidad y educación. Aportación al estado del arte en Etnomatemáticas
}

\author{
Juan Luis Piñeiro, Belén Giacomone y María Luisa Oliveras. Universidad de Granada
}

Recepción: 9 de junio de 2016 | Revisión: 30 de junio de 2016 | Aceptado: 12 julio de 2016

Correspondencia: oliveras@ugr.es

Citar: Piñeiro, J.L., Giacomone, B. y Oliveras, M.L. (2016). Interculturalidad y educación. Aportación al estado del arte en Etnomatemáticas. ReiDoCrea, 5, 161-165.

\begin{abstract}
Resumen: Las investigaciones crecientes en el área de la Etnomatemática han generado un amplio escenario de discusión en el campo de la educación matemática, y por consiguiente es posible observar un gran número de aportes a nivel mundial. En este sentido, se plantea la necesidad de indagar sobre cuáles son los aspectos principales que preocupan en este campo de la educación y contribuir de esta manera, al estado del arte sobre la Etnomatemática, y por consiguiente a la educación matemática y la diversidad cultural. Para afrontar este problema, se realizó en primer lugar, una búsqueda sistemática de publicaciones científicas, en actas de congresos y revistas de impacto durante el periodo 2014-2015, de la cual se obtuvieron 48 documentos vinculados al área de la etnomatemática de manera explícita o implícita. En segundo lugar, se analizó la muestra teniendo en cuenta el modelo MOMUME de Oliveras (2000, 2008a, 2008b). Esta herramienta metodológica nos permitió reflexionar sobre las tendencias que se están configurando en Etnomatemática y contribuir al estado del arte en este programa. Los resultados muestran tendencia a tratar temas tales como: sociedad, cultura y modelo educativo, a través de experimentos de campo y con metodologías cualitativas, fundamentados en las matemáticas como un constructo social y cultural.
\end{abstract}

Palabras clave: Multiculturalismo | Educación

Interculturalism and education. Contribution to the state of the art on Ethnomathematics

\begin{abstract}
Increasing researches in the Ethnomathematics area have generated a broad discussion's scenario in the field of mathematics education, and therefore, observing a large number of contributions worldwide is a fact. In this sense, the need to investigate about what main aspects are of concern in this field of education is considered, and thus contribute the state of the art on Ethnomathematics, and consequently on mathematics education and cultural diversity. To address this problem, first of all a systematic search of scientific publications from conference proceedings and journals with impact factor during the 2014-2015 period was performed; 48 documents related to Ethnomathematics area, explicitly or implicitly, were obtained as a sample from search. Secondly, the sample was analyzed according to the Oliveras's MO.MU.ME model (2000, 2008a, 2008b). This methodological tool allowed us to reflect about kind of trends that are setting up in Ethnomathematics and contribute to its state of art. The results show a tendency to deal with issues such as society, culture, and educational model, through field experiments and qualitative methodologies, based on mathematics as a social and cultural construct.
\end{abstract}

Keywords: Multiculturalism | Education

\section{Introducción}

Consideramos que indagar, sobre la naturaleza, la producción y trasmisión de los conocimientos matemáticos es una tarea importante a la que quisiéramos realizar un pequeño aporte. Nos posicionamos desde una concepción que entiende nuestra realidad de una manera diversa y plural, no objetiva o única, creemos en una realidad que se constituye colectivamente en torno a comunidades que construyen los saberes (Oliveras, 2006). Por tanto, entendemos las matemáticas como unas "multimatemáticas vivas", en el sentido en que Oliveras (2014) las describe, vinculando aspectos epistemológicos, sociológicos y psicológicos, como un trébol de tres hojas unidas por un mismo eje: con una hoja que las caracteriza como ciencia, otra hoja que indica que son una manifestación cultural inseparable del resto de los aspectos de cada cultura y la tercera hoja simboliza que son parte de nuestra forma de pensar y de concebir el mundo. (p.17) 
Para los investigadores es básico el trabajo difundido por publicaciones que transmiten los hallazgos y/o antecedentes sobre el estudio de un determinado tema, por tal motivo, desde estos constructos teóricos, nuestro trabajo pretende: a) organizar y analizar una muestra de publicaciones etnomatemáticas del período 2014/2015, y b) esbozar las tendencias de la producción sobre interculturalidad en la educación y aspectos críticos en el programa etnomatemático, realizando esta tarea mediante el modelo MOMUME (Oliveras, 2008a, 2008b).

Estos objetivos nos permitirían caracterizar, de alguna forma, las publicaciones sobre acciones etnomatemáticas realizadas en dicho período con el fin de continuar con la investigación bibliográfica de Albizu (2014), en la que se organizó y caracterizó el trabajo etnomatemático de años anteriores; asimismo, permitirán ampliar nuestros primeros hallazgos (sobre las publicaciones seleccionadas de las actas del PME38; Oliveras et al., 2015) avanzando hacia una construcción sistematizada del estado del arte sobre la educación matemática planteada desde una mirada sociopolítica, dentro de las que se encuentra la etnomatemática.

\section{Método}

El presente trabajo de análisis bibliográfico toma forma a partir de la búsqueda de publicaciones científicas, en actas y revistas de impacto, especializadas en educación matemática y algunas en etnomatemática (Sierra, 2011; Toerner y Arzarello, 2012). Se obtuvieron 48 documentos que fueron consideradas producciones significativas en el campo de las Etnomatemáticas. Para analizar dicha muestra tomamos los planteamientos teóricos y el modelo MO.MU.ME. [Model for the Study of Multiculturality in Mathematics Education] de Oliveras (2000, 2008a, 2008b). Esta herramienta metodológica nos permitió reflexionar sobre las tendencias que se están configurando dentro de las Etnomatemáticas. Se han realizado análisis cuantitativo y cualitativo de los datos tomados sobre los documentos que forman la muestra. Para ello, nuestro trabajo utiliza las herramientas proporcionadas por el citado modelo, constructo metodológico que surge como una propuesta específica de clasificación y análisis de la producción investigadora en la línea sociocultural y etnomatemática, utilizaremos sus instrumentos para organizar, analizar y sistematizar los datos de nuestra búsqueda.

\section{Muestra y contexto}

El presente trabajo emerge desde la actividad final del curso Etnomatemáticas del Máster en Didáctica de la Matemática de la Universidad de Granada. En esta asignatura hemos realizado una revisión bibliográfica en revistas catalogadas como de impacto (Sierra, 2011; Toerner y Arzarello, 2012), otras relativas a la etnomatemática y actas de congresos reconocidos en la comunidad científica. La muestra, finalmente, está formada por 48 documentos, localizados a partir de bases de datos, que fueron identificados mediante palabras claves previamente seleccionadas. El motivo de esta búsqueda surge ante la necesidad de encontrar toda la variedad de producciones en esta línea para potenciar nuevas investigaciones y difundir resultados ya obtenidos y que pueden ser aplicables en la educación, teniendo en cuenta que muchas veces estas investigaciones resultan desconocidas para profesores e investigadores situados fuera del ámbito etnomatemático. 


\section{Procedimiento}

La búsqueda fue realizada en las páginas web de las revistas especializadas y de los grupos responsables de las actas de los congresos. Una vez seleccionados los artículos, se construyó una lista por orden alfabético que posteriormente fue codificada (Anexo 1), usando una de las herramientas del modelo MOMUME. La búsqueda fue realizada en los lugares que se indican en la Tabla 1.

\begin{tabular}{|ll|}
\hline \multicolumn{2}{|c|}{ Tabla 1. Elementos de la búsqueda } \\
\hline Revistas: & Actas: \\
\hline -The mathematics enthusiast & -ICME (Congreso Internacional de Educación Matemática) Anual \\
-AIEM (Avances de Investigación en Educación & -SEIEM (Sociedad Española de Investigación en Educación \\
Matemática) Revista de la SEIEM & Matemática) \\
-Relime (Revista Latinoamericana de Investigación & -XV CEAM \\
en Matemática Educativa) & -PME-NA (Conference of the International Group for the Psychology \\
-Enseñanza de las Ciencias & of Mathematics Education y North American Chapter of the PME) \\
-Educational Studies in Mathematics & -ICEM (ISGEM Grupo Internacional de Estudio de Etnomatemáticas) \\
-Bolema (Boletim de Educação Matemática) & Palabras clave de búsqueda: \\
-ZDM Mathematics Education & Etnomatemáticas / Ethnomathematics \\
-The mathematics educator (TME) & Multiculturalidad / Multiculturalism \\
-Journal of Mathematics Teacher Education (JMTE) Educación intercultural / Intercultural Education \\
- RLE (Revista Latinoamericana de & Diversidad en matemáticas / Diversity in mathematics \\
Etnomatemáticas. Red latinoamericana de & Matemática crítica / Mathematical criticism \\
etnomatemática) & \\
\hline
\end{tabular}

\section{Instrumentos}

En este trabajo hemos utilizado los instrumentos del constructo MOMUME (Oliveras, 2008a, 2008b), para realizar un estudio sobre producciones científicas relativas a las etnomatemáticas durante el año 2014 hasta junio del 2015 y su posterior análisis. Estos instrumentos puestos a disposición de los investigadores consideran diferentes ideas. Dichas ideas y sus consideraciones son:

- Categorías fundamentales relativas a las áreas de investigación involucradas. Estas áreas de investigación son las matemáticas, la educación y la sociedad.

- Categorías instrumentales relativas a las actuaciones realizadas y sus fines. Se consideran las tipologías investigación básica, investigación de campo, experiencia de aula y estudio teórico.

- Subcategorías relativas al sujeto de estudio y al contenido del estudio. Son parte de las categorías instrumentales, llamadas por tanto, subcategorías instrumentales de orden 1 y de orden 2. Las primeras categorizan los trabajos a partir de las personas, grupos u objetos que se estudian y las acciones que se realizan en el proceso de investigación. Las segundas lo hacen atendiendo a los contenidos específicos que se abordan en ella.

- Sistemas de codificación y protocolos de organización de los documentos.

\section{Resultados}

EL análisis cualitativo ha permitido describir las temáticas utilizadas en las 48 publicaciones (Actas: 14=29,2\%; Artículos: 34=70,8\%; de ellos 11 en español=23\%) dentro de las correspondientes Categorías fundamentales (Tabla 2). Los resultados 
muestran una tendencia significativa de trabajos que aluden a temas como Sociedad (entendida como multicultural, multilingüe, consumidora y creadora de matemáticas), Cultura (que determina los modelos cognitivos, las motivaciones afectivas y las estructuras sociales) y Modelos Educativos (críticos, que construyan los saberes de forma inclusiva con todos los grupos culturales); contrariamente, los contenidos matemáticos concernientes al aula, el currículo y recursos materiales son los menos tratados. Cabe destacar que varios de estos conceptos se superponen en las publicaciones analizadas, del mismo modo que las categorías fundamentales no son excluyentes.

\begin{tabular}{|c|c|c|c|}
\hline \multicolumn{4}{|c|}{ Tabla 2. Categorías Fundamentales } \\
\hline & Categorías fundamentales & Cantidad & $\%$ \\
\hline Cultura & & 43 & 89,58 \\
\hline \multirow{2}{*}{ Matemáticas } & Epistemología, creencias, actitudes & 34 & 70,83 \\
\hline & Contenidos o temáticas matemáticas & 14 & 29,17 \\
\hline \multirow{2}{*}{ Educación } & Modelo educativo & 40 & 83,33 \\
\hline & Tipo de currículo, recursos matemáticos, otros. & 24 & 50 \\
\hline Sociedad & & 48 & 100 \\
\hline
\end{tabular}

Por otra parte, el análisis ha permitido clasificar dichas investigaciones dentro de las Categorías instrumentales (Tabla 3). En concordancia con los datos de Oliveras et al. (2015) se mantiene la tendencia de realizar mayoritariamente investigaciones de campo, seguida de la realización de estudios teóricos.

\begin{tabular}{|lccc|}
\hline \multicolumn{3}{c|}{ Tabla 3. Categorías instrumentales } & \\
\hline & Categorías Instrumentales & Cantidad & $\%$ \\
\cline { 2 - 4 } A. Investigaciones & A.I. Investigaciones de campo & 28 & $\mathbf{5 8 , 3}$ \\
\cline { 2 - 4 } & A.II. Investigaciones Básicas & 6 & 12,5 \\
\hline B. Experiencias & & 4 & 8,3 \\
\hline C. Estudios Teóricos & & 10 & 20,83 \\
\hline
\end{tabular}

Estos resultados nos permiten distinguir un creciente interés de la comunidad científica, en publicar trabajos relacionados con la diversidad cultural en el ambiente matemático escolar. De la misma manera, nos permiten observar las relaciones que tienen estas concepciones científicas con las investigaciones de campo, ya que son las que prevalecen en esta muestra analizada. Esto nos lleva a reflexionar sobre la importancia de hacer vivir a las matemáticas en un mundo culturalmente diverso y la necesidad de incidir efectivamente en la práctica educativa.

\section{Discusión y conclusiones}

A lo largo de este trabajo hemos logrado sintetizar los resultados obtenidos tras el análisis y la clasificación de 48 documentos en el campo de la etnomatemática, durante el periodo 2014-2015. Si bien en la búsqueda no se tuvieron en cuenta tesis científicas ni libros específicos del área, hemos centrado nuestro análisis en revistas y actas consideradas de gran impacto en la cultura educativa. Asimismo, el modelo MOMUME (Oliveras, 2008a, $2008 \mathrm{~b})$ nos ha permitido llevar a cabo el segundo objetivo planteado, mostrando las distintas tendencias con las que se aproximan las investigaciones a los diferentes aspectos de la interculturalidad en la educación matemática 
Las publicaciones que conformaron la muestra se caracterizan en general por tener una metodología de investigación cualitativa (estudio de casos, etnografía, análisis interpretativos). El idioma mayoritario de las publicaciones es el inglés seguido a partes iguales por el portugués y el español. Entre las 48 investigaciones analizadas, encontramos que los estudiantes de educación obligatoria de diversos continentes son los sujetos de estudio de interés, seguidos de los profesores (en formación inicial y en activo) y finalmente los artesanos. Destacan a la vez, las acciones puntuales en formación de estudiantes y profesores, seguidas de acciones de enseñanza formal reglada (ya sea en docencia, recursos didácticos o formación de profesores) y desarrollo de algunos proyectos didácticos. Este último resultado es destacado por Gavarrete (2013) en su trabajo sobre la evolución de la etnomatemática como campo de investigación, esto se debe a la necesidad de promover "el principio de alteridad cultural, que oriente hacia nuevas estrategias de acción educativa, con la que no se amenace las raíces ancestrales de los pueblos ni tampoco los limite o excluya dentro del panorama mundial" (p. 145).

Para finalizar, consideramos que la revisión bibliográfica realizada aporta resultados razonables hacia la ampliación del estado del arte y aporta un medio "para incidir en la toma de conciencia crítica sobre las matemáticas y su valor social" (Gavarrete, 2013, p. 145). La utilización del modelo MOMUME nos entrega una visión general sobre las temáticas relevantes para los investigadores y el tipo de estudio. Dichas tendencias están tratando de dilucidar una concepción de las matemáticas, que a nuestro parecer, tiene estrecha relación con la metáfora del trébol, utilizada por Oliveras (2000) para definir las matemáticas, cada vez más consideradas como un constructo social y cultural que ayuda a comprender el mundo (Bishop, 1999).

\section{Referencias}

Albizu, U. (2014). Bases para la investigación y la práctica educativa desde las etnomatemáticas (Tesis de maestría no publicada). Universidad de Granada, España.

Bishop A. (1999). Enculturación matemática. La educación matemática desde una perspectiva cultural. Barcelona: Paidós.

Gavarrete, M. E. (2013). La Etnomatemática como campo de investigación y acción didáctica: su evolución y recursos para la formación de profesores desde la equidad. Revista Latinoamericana de Etnomatemática, 6(1), 127-149.

Oliveras, M. L. (2000). Matemáticas. En J. Fuentes y M. L. Oliveras (Eds.), Matemáticas en la Sociedad (pp. 13-27). Granada, España: Repro-digital Constitución.

Oliveras, M. L. (2006). Etnomatemáticas: de la multiculturalidad al mestizaje. Microproyectos integrados para educación matemática intercultural. En J. Goñi, N. Gorgorió, M. Prat, M. Santesteban, G. Inés, C. Nunes, ... R. Díaz (Eds.), Matemáticas e Interculturalidad (pp. 117 - 149). Barcelona, España: Graó.

Oliveras, M. L. (2008a). Model for research on Multiculturality in Mathematics Education. En M. L. Oliveras y N. de Bengoechea (Eds.), ICME 11, Topic Study Group 33: Mathematics education in a multilingual and multicultural environment. Monterrey, Mexico.

Oliveras, M. L. (2008b). Study of «the state of the question about Multiculturality and Mathematics Education». En M. L. Oliveras \& N. de Bengoechea (Eds.), ICME 11, Topic Study Group 33: Mathematics education in a multilingual and multicultural environment. Monterrey, México.

Oliveras, M. L. (2014). Etnomatemática: El vértice matemático del posmodernismo poliédrico. Journal of Mathematics y Culture Special Edition: Proceedings of the 5th International Congress on Ethnomathematics - ICEm5, 8(1), 94-96.

Oliveras, M. L., Giacomone, B. y Piñeiro, J. L. (2015). Etnomatemáticas en congresos internacionales. Aportación al estado del arte sobre la educación matemática y la diversidad cultural. ReiDoCrea, (Monográfico 2), 13-21. 
Toerner, G. y Arzarello, F. (2012). Grading mathematics education research journals. EMS News Letters, (86), 52-54. 